DOI: 10.1002/ejoc.201301283

\title{
A Convenient Route for the Synthesis of 3-Deazaspongosine
}

\author{
Omprakash Bande ${ }^{[a]}$ and Piet Herdewijn*[a]
}

Keywords: Ribonucleosides / Nitrogen heterocycles / Glycosylation / Nucleobases / Nucleosides / Aromatic substitution

The first chemical synthesis of the 3-deazaspongosine nucleoside is described, starting from commercially available 4amino-2,6-dichloropyridine. The key step is the introduction of required functional groups at the 2 and 6 positions of the 4-amino-3-nitropyridine without any conflict in the synthesis of nucleobase. Regioselective nucleophilic substitution with allyl alkoxide at the 2 position of 4-amino-2,6-dimethoxypyridine, followed by sequential deallylation and chlorination led to the desired 2-chloro derivative. Ring closure of the 3,4diaminopyridine and stereoselective glycosylation of the imidazo[4,5-c]pyridine with tetraacetate-protected ribofuranose gave only the $N^{9}-\beta$-isomer. A final nucleophilic displacement of the 6-chloride by hydrazine followed by reduction with Raney Nickel gave the desired 3-deazaspongosine.

\section{Introduction}

There is much evidence in the scientific literature that modification of the nucleobase and/or the sugar moiety of naturally occurring purine nucleosides can result in analogues that show interesting biological effects. ${ }^{[1-5]}$ The nitrogen atoms in purine nucleosides are of particular interest, as they are important for binding to enzymes. ${ }^{[6,7]} 3$-Deazapurine nucleosides deserve extra attention, because $\mathrm{N}^{3}$ of purine nucleosides is considered to be involved in stabilizing the syn conformation by intramolecular hydrogen bonding $\left(5^{\prime}-\mathrm{OH}-\mathrm{N}^{3} \mathrm{H}\right){ }^{\left[{ }^{8]}\right.} \mathrm{A}$ literature survey revealed that several 3deazapurine ${ }^{[9,10]}$ nucleosides and nucleotides have been synthesized, and that these compounds have been found to have antibacterial, ${ }^{[11]}$ antiviral, ${ }^{[12,13]}$ immunosuppressive, ${ }^{[14]}$ anti-inflammatory, ${ }^{[15]}$ and anticancer activities. ${ }^{[16]}$ Thus, we aimed to synthesize the 3-deaza analogue of spongosine nucleoside 1 (Figure 1).
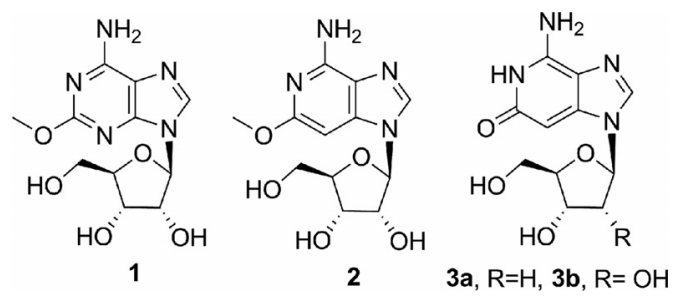

Figure 1. Structures of spongosine, 3-deazaspongosine, and 3-deazaisoguanosine.

[a] Medicinal Chemistry, Rega Institute for Medical Research, KU Leuven,

Minderbroedersstraat 10, 3000 Leuven, Belgium E-mail: Piet.Herdewijn@rega.kuleuven.be http://medchem.rega.kuleuven.ac.be/

Supporting information for this article is available on the WWW under http://dx.doi.org/10.1002/ejoc.201301283.
2-Alkoxyadenosine is a purine nucleoside comprising a 2 -alkoxyadenine unit attached to a ribose moiety by a $\beta$ $N^{9}$-glycosidic bond. 2-Alkoxyadenosines are well known for their biological activities, for example, as potent and selective agonists of the coronary artery A2 adenosine receptor, ${ }^{[17]}$ and as antibacterial adenosine LigA inhibitors. ${ }^{[18,19]}$ One such compound, 2-methoxyadenosine (1, Figure 1), commonly known as spongosine, ${ }^{[20,21]}$ which was isolated from the Caribbean sponge Cryptotethia crypta, has been shown to have antiviral activity ${ }^{[22]}$ and antiplatelet properties. ${ }^{[23]}$ It is also used in the treatment of B-cell proliferative diseases $^{[24]}$ and in the treatment of pain. ${ }^{[25]}$ Spongosine was not only the first methoxy purine to be found in nature, but also one of the first $O$-methyl compounds to be isolated from animal tissues. ${ }^{[26]}$

3-Deazaspongosine (2) could be a synthon for the synthesis of 3-deazaisoguanosine (3b), which could be considered to be a new nonstandard nucleoside. ${ }^{[27,28]}$ Despite the remarkable properties of spongosine as described above, to date, no strategy for the synthesis of its 3-deaza analogues has been reported. Only its 8-aza analogue has been synthesized by Kobe et al. ${ }^{[29,30]}$ As part of our continuing research in nucleoside chemistry, ${ }^{[31,32]}$ in this paper, we report the first preparation of the hitherto unknown 3-deazaspongosine nucleoside 2.

In our retrosynthetic planning, we proposed three synthetic routes (Scheme 1). In proposed route A, 3-deazaspongosine (2) could be obtained from nucleoside 7 by ammonolysis of the chloride at the 2 position, followed by nucleophilic substitution of second chloride. Nucleoside 7 could be synthesized by coupling 2,6-dichloro-imidazo[4,5c]pyridine (6) with tetraacetate-protected ribofuranose in the presence of a Lewis acid. Compound 6 could be obtained from 4-amino-2,6-dichloro-3-nitropyridine (5) by reduction of the nitro group followed by ring closure. In pro- 
posed route B, compound 2 could be prepared from nucleoside 13 by displacement of the methoxy group at the 4 position using an ammonia source. Nucleoside $\mathbf{1 3}$ could be synthesized by performing a glycosylation between intermediate $\mathbf{1 2}$ and tetraacetate-protected ribofuranose in the presence of a Lewis acid. Imidazo[4,5-c]pyridine $\mathbf{1 2}$ could be synthesized from 4-amino-2,6-dimethoxy-3-nitropyridine (10) by reduction of the nitro group followed by ring closure. In route $\mathrm{C}$, compound $\mathbf{2}$ could be prepared from nucleoside 19 by nucleophilic substitution of the 2-chloride by an ammonia source. Nucleoside 19 could be synthesized by coupling of 4-chloro-6-methoxyimidazo[4,5-c]pyridine (18) with tetraacetate-protected ribofuranose in the presence of a Lewis acid. Imidazopyridine $\mathbf{1 8}$ could be synthesized from 4-amino-2-chloro-6-methoxy-3-nitropyridine $\mathbf{1 7}$ by reduction of the nitro group followed by ring closure, as shown in Scheme 1.

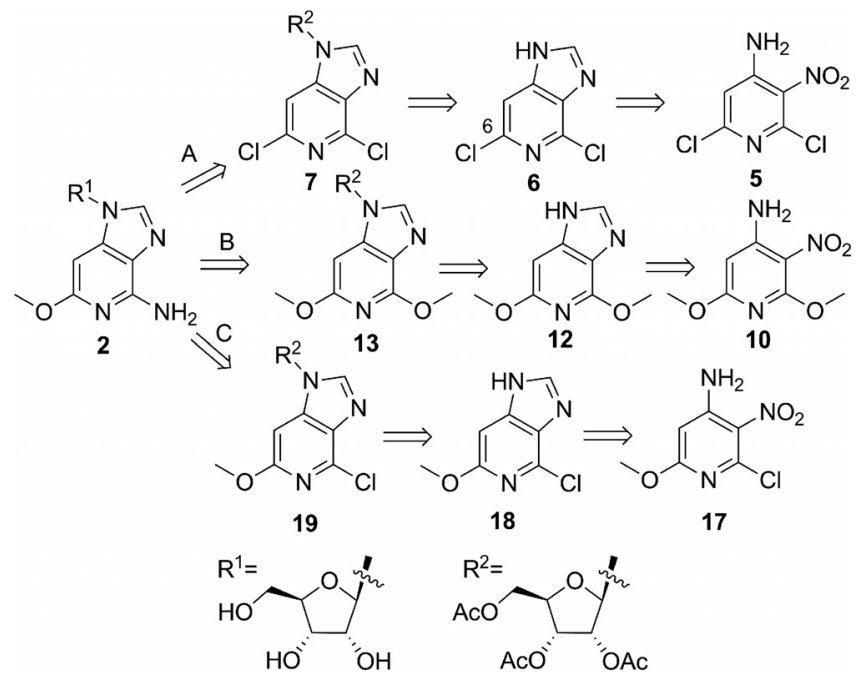

Scheme 1. Retrosynthetic analysis of 3-deazaspongosine (2).

\section{Results and Discussion}

The required 2,6-dichloro-3-nitropyridin-4-amine (5) was prepared from commercially available 4-amino-2,6-dichloropyridine (4) as reported by Cosstick et al. (Scheme 2). ${ }^{[33]}$ Reduction of the nitro group of compound $\mathbf{5}^{[34,35]}$ gave 3,4-diaminopyridine, which was used without purification in the ring closure reaction. Thus, the crude material was heated at reflux in triethyl orthoformate and then in formic acid to give 4,6-dichloro-imidazo[4,5-c]pyridine (6) in $72 \%$ yield over three steps. Coupling of nucleobase 6 with tetraacetate-protected ribofuranose 9 in the presence of $p$-toluenesulfonic acid gave nucleoside $\mathbf{7}$ in good yield, as reported by Montgomery et al. ${ }^{[36]}$ Treatment of derivative 7 with ammonia ( $30 \%$ aq.) at $200{ }^{\circ} \mathrm{C}$ for $5 \mathrm{~d}$ resulted in regioselective displacement of the 2-chloride and concomitant deacetylation to give compound $\mathbf{8}$ ( $80 \%$ yield). The spectral and analytical data of $\mathbf{8}$ were found to be consistent with those reported. ${ }^{[36]}$ Unfortunately, all our attempts to displace the second chloride of imidazo[4,5-c]pyridine $\mathbf{8}$ using sodium methoxide or palladium-catalyzed cross-coupling reactions ${ }^{[37]}$ failed.

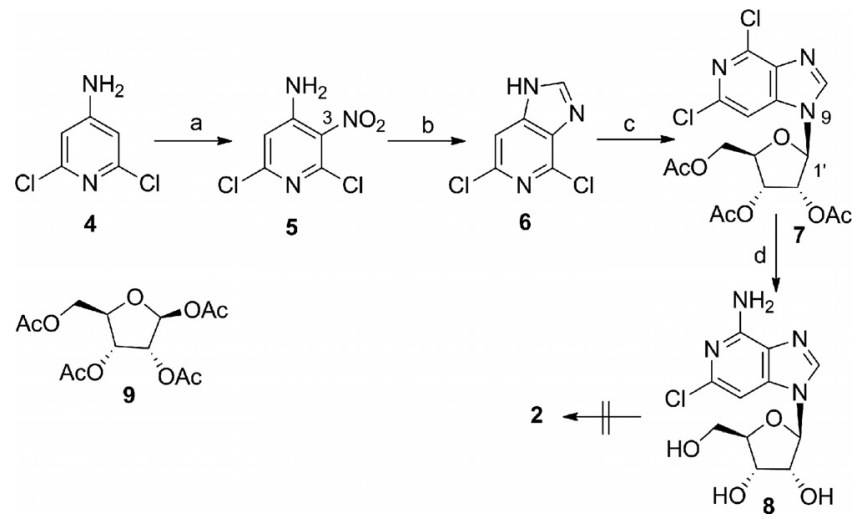

Scheme 2. Reagents and conditions: (a) ref. ${ }^{[33]}$; (b) (i) $\mathrm{Fe}, \mathrm{HCl}$, EtOH; (ii) triethyl orthoformate, reflux; (iii) formic acid, reflux, $72 \%$; (c) 9, p-toluenesulfonic acid, $160{ }^{\circ} \mathrm{C}, 10 \mathrm{~min}, 76 \%$; (d) $\mathrm{NH}_{3}$ (30\% aq.), $200{ }^{\circ} \mathrm{C}, 5 \mathrm{~d}, 80 \%$.

As our attempts to displace the second chloride in intermediate $\mathbf{8}$ were unsuccessful, we decided to perform a nucleophilic substitution at an earlier stage, with a more reactive starting material such as $\mathbf{5}$. Treatment of pyridine $\mathbf{5}$ with a solution of sodium methoxide in methanol at $60{ }^{\circ} \mathrm{C}$ for $12 \mathrm{~h}$ led to the desired disubstituted derivative (i.e., 10) in $90 \%$ yield (Scheme 3). Reduction of the nitro group was performed by treatment with sodium dithionite at room temperature for $15 \mathrm{~min}$ to give 3,4-diamino derivative $\mathbf{1 1}$ in $75 \%$ yield. Subsequent ring closure with triethyl orthoformate and formic acid gave 4,6-dimethoxy-imidazo[4,5-c]pyridine (12) in $85 \%$ yield. Glycosylation of $\mathbf{1 2}$ with tetraacetate-protected ribofuranose 9 using DBU (1,8-diazabicyclo[5.4.0]undec-7-ene) and TMSOTf (trimethylsilyl trifluoromethanesulfonate) gave a mixture of $N^{9}$ (13a) and $N^{7}$ (13b) nucleoside isomers in a 7:3 ratio in $82 \%$ yield. The structure of the $N^{9}$ and $N^{7}$ isomers was confirmed by HMBC spectral analysis. When we attempted to displace the methoxy group by an $\mathrm{NH}_{2}$ group, we only observed

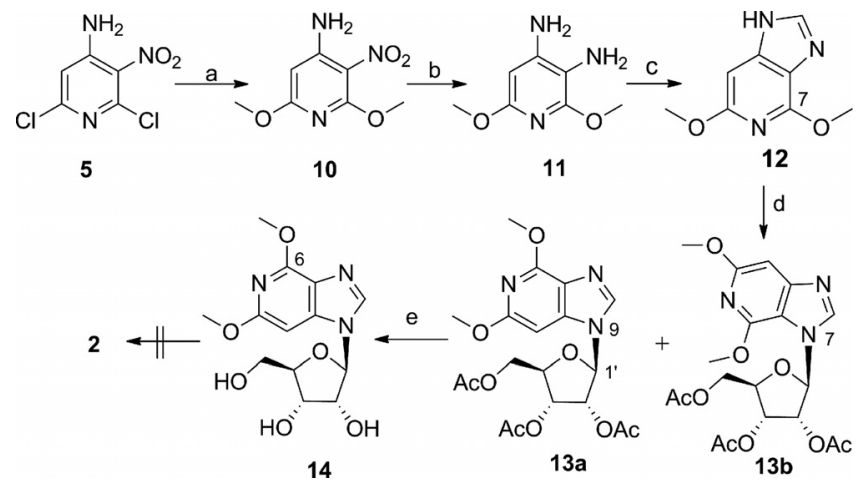

Scheme 3. Reagents and conditions: (a) $\mathrm{NaOMe}, \mathrm{MeOH}, 12 \mathrm{~h}$, $60{ }^{\circ} \mathrm{C}, 90 \%$; (b) (i) $\mathrm{Na}_{2} \mathrm{~S}_{2} \mathrm{O}_{4}, \mathrm{EtOH} / \mathrm{H}_{2} \mathrm{O}$ (1:1), room temp., 15 min, $75 \%$; (c) (i) triethyl orthoformate, reflux, $5 \mathrm{~h}$; (ii) formic acid, reflux, $5 \mathrm{~h}, 85 \%$; (d) 9, TMSOTf, DBU, $\mathrm{CH}_{3} \mathrm{CN}, 0{ }^{\circ} \mathrm{C}$ to $65^{\circ} \mathrm{C}, 12 \mathrm{~h}$; (e) $\mathrm{NH}_{2} \mathrm{NH}_{2}, \mathrm{EtOH}, 100{ }^{\circ} \mathrm{C}, 12 \mathrm{~h}, 95 \%$. 
cleavage of the protective groups to give derivative 14. A number of attempts were made using ammonia (methanolic or aqueous solutions) in a sealed tube, ammonia/CuI ${ }^{[38]}$ or hydrazine $(85 \%)$, but none of these methods led to the desired 3-deazaspongosine.

As an alternative synthetic route, the regioselective addition of allyl alcohol was achieved by treating $\mathbf{1 0}$ with a solution of sodium allyl alkoxide ( $5 \mathrm{~m}$ in allyl alcohol) at $60{ }^{\circ} \mathrm{C}$ for $1.5 \mathrm{~h}$ to give monosubstituted derivative $\mathbf{1 5}$ in $82 \%$ yield (Scheme 4 ). Deallylation of $\mathbf{1 5}$ was accomplished by treatment with palladium(0) and 1,3-dimethylbarbituric acid at room temperature in anhydrous dichloromethane to give hydroxy derivative $\mathbf{1 6}$ in $98 \%$ yield. ${ }^{[39]}$ Compound $\mathbf{1 6}$ was heated at reflux in $\mathrm{POCl}_{3}$ for $2.5 \mathrm{~h}$ to give chlorinated intermediate $\mathbf{1 7}$ in $78 \%$ yield. The synthesis of imidazo[4,5c]pyridine $\mathbf{1 8}$ was performed in three sequential steps. First, the nitro group was reduced by treatment with sodium dithionite at room temperature for $15 \mathrm{~min}$, then ring closure was achieved by refluxing successively in triethyl orthoformate and formic acid. Stereoselective glycosylation was carried out using DBU and TMSOTf to give the $N^{9}-\beta$-isomer only. An attempted one-pot nucleophilic displacement and removal of the acetate groups by treatment with ammonia solution at $160{ }^{\circ} \mathrm{C}$ led to the decomposition of nucleoside 19. We thus envisioned that the deprotection and nucleophilic substitution could be achieved by treatment with hydrazine. In fact, when nucleoside 19 was treated with an aqueous solution of hydrazine $(80 \%)$ at $100{ }^{\circ} \mathrm{C}$ for $2 \mathrm{~h}$, followed by an additional step with Raney Nickel at $100{ }^{\circ} \mathrm{C}$ for $1.5 \mathrm{~h}, 3$-deazaspongosine (2) was formed. The structure of $N^{9}$ isomer 2 was confirmed by HMBC analysis (correlation between $\mathrm{C}-4$ and $1^{\prime}-\mathrm{H}$ ).

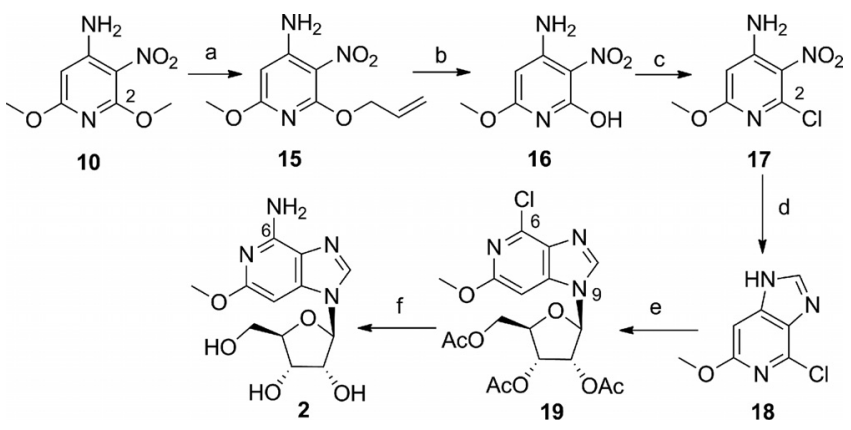

Scheme 4. Reagents and conditions: (a) sodium allylate, allyl alcohol, $60{ }^{\circ} \mathrm{C}, 1.5 \mathrm{~h}, 82 \%$; (b) $\mathrm{Pd}\left(\mathrm{PPh}_{3}\right)_{4}, 1,3$-dimethylbarbituric acid, $\mathrm{CH}_{2} \mathrm{Cl}_{2}$, room temp., $2.5 \mathrm{~h}, 98 \%$; (c) $\mathrm{POCl}_{3}$, reflux, $2.5 \mathrm{~h}$, $78 \%$; (d) (i) $\mathrm{Na}_{2} \mathrm{~S}_{2} \mathrm{O}_{4}, \mathrm{EtOH} / \mathrm{H}_{2} \mathrm{O}$ (1:1), room temp., 15 min. (ii) triethyl orthoformate, reflux, $5 \mathrm{~h}$; (iii) formic acid, reflux, $5 \mathrm{~h}, 68 \%$; (e) 9, TMSOTf, DBU, $\mathrm{CH}_{3} \mathrm{CN}, 65^{\circ} \mathrm{C}, 12 \mathrm{~h}, 74 \%$; (f) (i) $80 \%$ $\mathrm{NH}_{2} \mathrm{NH}_{2}, \mathrm{EtOH}, 100{ }^{\circ} \mathrm{C}, 2 \mathrm{~h}$; (ii) Raney $\mathrm{Ni}$ (50\% aq.), EtOH, $100{ }^{\circ} \mathrm{C}, 1.5 \mathrm{~h}, 65 \%$.

\section{Conclusions}

In conclusion, we have developed an efficient route for the synthesis of 3-deazaspongosine (2) starting from 2,6dichloro-4-amino-pyridine (4). The key steps were the selective introduction/removal of allyl alkoxide group, and the chlorination leading to $\mathbf{1 7}$. Stereoselective glycosylation of imidazo[4,5-c]pyridine 18 with tetraacetate-protected ribofuranose 9 yielded only the $N^{9}-\beta$-isomer 19 in good yield. A final nucleophilic displacement with hydrazine and reduction with Raney Nickel led to 3-deazaspongosine (2). This approach is general, as it is useful for the synthesis of a series of 2-alkoxyadenosine derivatives.

\section{Experimental Section}

General Remarks: ${ }^{1} \mathrm{H}$ and ${ }^{13} \mathrm{C}$ NMR spectra were recorded with $300 \mathrm{MHz}\left({ }^{1} \mathrm{H}, 300 \mathrm{MHz} ;{ }^{13} \mathrm{C}, 75 \mathrm{MHz}\right), 500 \mathrm{MHz}\left({ }^{1} \mathrm{H}, 500 \mathrm{MHz}\right.$; $\left.{ }^{13} \mathrm{C}, 125 \mathrm{MHz}\right)$, or $600 \mathrm{MHz}\left({ }^{1} \mathrm{H}, 600 \mathrm{MHz} ;{ }^{13} \mathrm{C}, 150 \mathrm{MHz}\right)$ spectrometers. 2D NMR spectra (H,H-COSY, HSQC, and HMBC) were used for the assignment of all the intermediate and final compounds. Mass spectra were acquired with a quadrupole orthogonal acceleration time-of-flight mass spectrometer. Melting points were obtained with a melting point apparatus using open capillary tubes. Column chromatographic separations were carried out on silica gel (100-200 mesh or $230-400$ mesh) by gradient elution with combinations of two/three solvents. Solvents for reactions were distilled before use: THF and toluene from Na/benzophenone; $\mathrm{CH}_{2} \mathrm{Cl}_{2}$ and $\mathrm{CH}_{3} \mathrm{CN}$ from $\mathrm{CaH}_{2} ; \mathrm{Et}_{3} \mathrm{~N}$ and pyridine from $\mathrm{KOH}$.

4,6-Dichloro-1 $\boldsymbol{H}$-imidazo[4,5-c]pyridine (6): Iron powder $(1.3 \mathrm{~g}$, $24.04 \mathrm{mmol}), \mathrm{H}_{2} \mathrm{O}(4.3 \mathrm{~mL}, 240 \mathrm{mmol})$, and $\mathrm{HCl}$ (concentrated; $1 \mathrm{~mL}, 28.85 \mathrm{mmol}$ ) were added to 4-amino-2,6-dichloro-3-nitropyridine $(5 ; 1 \mathrm{~g}, 4.81 \mathrm{mmol})$ in ethanol $(23 \mathrm{~mL})$. The mixture was heated at reflux for $16 \mathrm{~h}$. The mixture was cooled to room temperature, then it was neutralized with sodium hydrogen carbonate (saturated aq.). The mixture was filtered, and the filter residue was washed with ethyl acetate. The filtrate was concentrated. The residue was dissolved in ethyl acetate and washed with water $(10 \mathrm{~mL})$. The organic layer was dried $\left(\mathrm{MgSO}_{4}\right)$ and evaporated. The solid residue was used in the next step without further purification.

A mixture of 3,4-diaminopyridine $(500 \mathrm{mg}, 22.81 \mathrm{mmol})$ and triethyl orthoformate $(6.5 \mathrm{~mL}, 39.32 \mathrm{mmol})$ was heated at reflux at $145^{\circ} \mathrm{C}$ for $5 \mathrm{~h}$. The solution was then evaporated to dryness using a rotary evaporator. The residue was dissolved in formic acid $(99 \%$; $6.3 \mathrm{~mL}$ ), and the solution was heated at reflux at $110^{\circ} \mathrm{C}$ for $5 \mathrm{~h}$. When the reaction was complete, the excess formic acid was removed with the rotary evaporator. The residue was purified by column chromatography (dichloromethane/methanol, 97:3) to give 6 (390 mg, 72\%) as a yellow solid. $R_{\mathrm{f}}=0.65$ (dichloromethane/methanol, 9:1). m.p. $252-254{ }^{\circ} \mathrm{C} .{ }^{1} \mathrm{H}$ NMR (300 MHz, [D $]$ DMSO): $\delta=$ 8.54 (s, $1 \mathrm{H}, 2-\mathrm{H}), 7.75$ (s, $1 \mathrm{H}, 7-\mathrm{H}) \mathrm{ppm} .{ }^{13} \mathrm{C}$ NMR $(75 \mathrm{MHz}$, [D $]$ DMSO): $\delta=146.3$ (C-2), 143.4, 139.6, 137.6, 135.5 (C-6, C-4, C-3a, C-7a), 108.3 (C-7) ppm. HRMS (ESI ${ }^{+}$): calcd. for $\mathrm{C}_{6} \mathrm{H}_{3} \mathrm{Cl}_{2} \mathrm{~N}_{3}$ $[\mathrm{M}+\mathrm{H}]^{+}$187.9776; found 187.9771 .

4,6-Dichloro-9-(2,3,5-tri- $O$-acetyl- $\beta$-D-ribofuranosyl)-3-deazapurine (7): Reaction of nucleobase 6 with tetraacetate-protected ribofuranose 9 in the presence of $p$-toluenesulfonic acid gave nucleoside 7, as reported by Montgomery et al. ${ }^{[36]}$ Spectral and analytical data were in agreement with previous reports. $R_{\mathrm{f}}=0.3$ ( $n$-hexane/ethyl acetate, 1:1). m.p. $240-242{ }^{\circ} \mathrm{C} .{ }^{1} \mathrm{H}$ NMR $\left(500 \mathrm{MHz},\left[\mathrm{D}_{6}\right] \mathrm{DMSO}\right)$ : $\delta=8.79(\mathrm{~s}, 1 \mathrm{H}, 8-\mathrm{H}), 8.03(\mathrm{~s}, 1 \mathrm{H}, 3-\mathrm{H}), 6.40(\mathrm{~d}, J=5.70 \mathrm{~Hz}, 1$ $\left.\mathrm{H}, 1^{\prime}-\mathrm{H}\right), 5.62\left(\mathrm{t}, J=5.70 \mathrm{~Hz}, 1 \mathrm{H}, 2^{\prime}-\mathrm{H}\right), 5.41-5.45\left(\mathrm{~m}, 1 \mathrm{H}, 3^{\prime}-\right.$ $\mathrm{H}), 4.31-4.50\left(\mathrm{~m}, 3 \mathrm{H}, 4^{\prime}-\mathrm{H}, 5 \mathrm{a}^{\prime}-\mathrm{H}, 5 \mathrm{~b}^{\prime}-\mathrm{H}\right), 2.13\left(\mathrm{~s}, 3 \mathrm{H}, \mathrm{CH}_{3}\right)$, 2.09 (s, $\left.3 \mathrm{H}, \mathrm{CH}_{3}\right), 2.04$ (s, $\left.3 \mathrm{H}, \mathrm{CH}_{3}\right)$ ppm. ${ }^{13} \mathrm{C} \mathrm{NMR}(125 \mathrm{MHz}$, [D $]$ DMSO): $\delta=170.1,169.5,169.4(3 \mathrm{C}=\mathrm{O}), 146.0$ (C-6), 141.8, 140.8, 139.6, 137.3 (C-2, C-4, C-8, C-5), 107.3 (C-3), 86.8 (C-1'), $79.9\left(\mathrm{C}-4^{\prime}\right), 72.2\left(\mathrm{C}-2^{\prime}\right), 69.4\left(\mathrm{C}-3^{\prime}\right), 62.9\left(\mathrm{C}-5^{\prime}\right), 20.6,20.4,20.2$ (3 
$\left.\mathrm{CH}_{3}\right)$ ppm. HRMS $\left(\mathrm{ESI}^{+}\right)$: calcd. for $\mathrm{C}_{17} \mathrm{H}_{17} \mathrm{Cl}_{2} \mathrm{~N}_{3} \mathrm{O}_{7}[\mathrm{M}+\mathrm{H}]^{+}$ 446.0503; found 446.0504.

2,6-Dichloro-9-( $\beta$-D-ribofuranosyl)-3-deazapurine (8): A solution of 13a $(300 \mathrm{mg}, 672 \mu \mathrm{mol})$ in ammonia $(30 \%$ aq.) was heated at $200{ }^{\circ} \mathrm{C}$ for $5 \mathrm{~d}$. The reaction mixture was concentrated, and the residue was purified by column chromatography (dichloromethane/ methanol, 9:1) to give $14(180 \mathrm{mg}, 93 \%)$ as a solid. $R_{\mathrm{f}}=0.5$ (dichloromethane/methanol, 9:1). m.p. $224-226{ }^{\circ} \mathrm{C} . \quad{ }^{1} \mathrm{H} \quad \mathrm{NMR}$ (600 MHz, CD $\left.\mathrm{CD}_{3} \mathrm{OD}\right): \delta=8.33$ (s, $\left.1 \mathrm{H}, 8-\mathrm{H}\right), 7.07$ (s, $\left.1 \mathrm{H}, 3-\mathrm{H}\right), 5.82$ $\left(\mathrm{d}, J=5.80 \mathrm{~Hz}, 1 \mathrm{H}, 1^{\prime}-\mathrm{H}\right), 4.38\left(\mathrm{t}, J=5.80 \mathrm{~Hz}, 1 \mathrm{H}, 2^{\prime}-\mathrm{H}\right), 4.25$ $\left(\mathrm{dd}, J=3.60,5.80 \mathrm{~Hz}, 1 \mathrm{H}, 3^{\prime}-\mathrm{H}\right), 4.11(\mathrm{dt}, J=3.60,6.0 \mathrm{~Hz}, 1 \mathrm{H}$, $\left.4^{\prime}-\mathrm{H}\right), 3.84\left(\mathrm{dd}, J=6.0,12.0 \mathrm{~Hz}, 1 \mathrm{H}, 5 \mathrm{a}^{\prime}-\mathrm{H}\right), 3.77(\mathrm{dd}, J=3.60$, $\left.12.0 \mathrm{~Hz}, 1 \mathrm{H}, 5 \mathrm{~b}^{\prime}-\mathrm{H}\right)$ ppm. ${ }^{13} \mathrm{C}$ NMR $\left(125 \mathrm{MHz}, \mathrm{CD}_{3} \mathrm{OD}\right): \delta=$ 152.5 (C-6), 143.2 (C-2), 142.1 (C-8), 141.1 (C-4), 126.8 (C-5), 97.8 (C-3), $91.0\left(\mathrm{C}-1^{\prime}\right), 87.3\left(\mathrm{C}-4^{\prime}\right), 76.1\left(\mathrm{C}-2^{\prime}\right), 71.7\left(\mathrm{C}-3^{\prime}\right), 62.5\left(\mathrm{C}-5^{\prime}\right)$ ppm. HRMS $\left(\mathrm{ESI}^{+}\right)$: calcd. for $\mathrm{C}_{11} \mathrm{H}_{13} \mathrm{ClN}_{4} \mathrm{O}_{4}[\mathrm{M}+\mathrm{H}]^{+} 301.0698$; found 301.0693 .

2,6-Dimethoxy-3-nitropyridin-4-amine (10): Sodium methoxide (5 M in $\mathrm{MeOH}$; $2.8 \mathrm{~mL}$ ) was added to a solution of 5 (500 mg, $2.4 \mathrm{mmol})$ in methanol $(14 \mathrm{~mL})$, and the mixture was heated at $60{ }^{\circ} \mathrm{C}$ for $12 \mathrm{~h}$. The mixture was concentrated, then the residue was dissolved in ethyl acetate and washed with water $(5 \mathrm{~mL})$. The solution was dried $\left(\mathrm{MgSO}_{4}\right)$ and concentrated, and the residue was purified by column chromatography ( $n$-hexane/ethyl acetate, 9:1) to give 12 (430 mg, $90 \%)$ as a yellow solid. $R_{\mathrm{f}}=0.25$ ( $n$-hexane/ethyl acetate, $\left.8: 2\right)$. m.p. 136-138 ${ }^{\circ} \mathrm{C} .{ }^{1} \mathrm{H}$ NMR $\left(500 \mathrm{MHz},\left[\mathrm{D}_{6}\right] \mathrm{DMSO}\right): \delta=7.35$ (br. s, 2 $\left.\mathrm{H}, \mathrm{NH}_{2}\right), 5.78(\mathrm{~s}, 1 \mathrm{H}, 5-\mathrm{H}), 3.89\left(\mathrm{~s}, 3 \mathrm{H}, \mathrm{OCH}_{3}\right), 3.81(\mathrm{~s}, 3 \mathrm{H}$, $\left.\mathrm{OCH}_{3}\right) \mathrm{ppm} .{ }^{13} \mathrm{C} \mathrm{NMR}\left(125 \mathrm{MHz},\left[\mathrm{D}_{6}\right] \mathrm{DMSO}\right): \delta=162.8(\mathrm{C}-6)$, 158.6 (C-2), 153.9 (C-4), 115.8 (C-3), 86.2 (C-5), 54.0, 53.7, (2 $\left.\mathrm{OCH}_{3}\right)$ ppm. HRMS $\left(\mathrm{ESI}^{+}\right)$: calcd. for $\mathrm{C}_{7} \mathrm{H}_{9} \mathrm{~N}_{3} \mathrm{O}_{4}[\mathrm{M}+\mathrm{H}]^{+}$ 200.0665; found 200.0670.

2,6-Dimethoxypyridine-3,4-diamine (11): A solution of sodium dithionite $(1.2 \mathrm{~g}, 7.53 \mathrm{mmol})$ in water $(12.5 \mathrm{~mL})$ was added to a solution of $10(500 \mathrm{mg}, 2.51 \mathrm{mmol})$ in ethanol $(12.5 \mathrm{~mL})$, and the mixture was stirred at room temperature for $15 \mathrm{~min}$. The ethanol was evaporated at $20^{\circ} \mathrm{C}$. The residue was dissolved in ethyl acetate and washed with water $(6 \mathrm{~mL})$. The organic layer was dried $\left(\mathrm{MgSO}_{4}\right)$, and concentrated. The residue was purified by column chromatography ( $n$-hexane/ethyl acetate, 1:1) to give $\mathbf{1 1}(320 \mathrm{mg}, 75 \%)$ as a yellow solid. $R_{\mathrm{f}}=0.3$ ( $n$-hexane/ethyl acetate, 1:1). m.p. $70-72{ }^{\circ} \mathrm{C}$. ${ }^{1} \mathrm{H}$ NMR (300 MHz, [D $]$ DMSO): $\delta=5.64$ (s, $\left.1 \mathrm{H}, 5-\mathrm{H}\right), 5.35$ (br. s, $2 \mathrm{H}, \mathrm{NH}_{2}$ ), 3.51 (br. s, $2 \mathrm{H}, \mathrm{NH}_{2}$ ), 3.79 (s, $\left.3 \mathrm{H}, \mathrm{OCH}_{3}\right), 3.67$ (s, $\left.3 \mathrm{H}, \mathrm{OCH}_{3}\right) \mathrm{ppm} .{ }^{13} \mathrm{C} \mathrm{NMR}\left(75 \mathrm{MHz},\left[\mathrm{D}_{6}\right] \mathrm{DMSO}\right): \delta=154.7$, 150.0 (C-6, C-2), 146.0 (C-4), 109.0 (C-3), 86.9 (C-5), 52.5, 52.4 (2 $\left.\mathrm{OCH}_{3}\right)$ ppm. HRMS $\left(\mathrm{ESI}^{+}\right)$: calcd. for $\mathrm{C}_{7} \mathrm{H}_{11} \mathrm{~N}_{3} \mathrm{O}_{2}[\mathrm{M}+\mathrm{H}]^{+}$ 170.0923; found 170.0927.

4,6-Dimethoxy-1H-imidazo[4,5-c]pyridine (12): A mixture of 3,4-diaminopyridine $\mathbf{1 1}(600 \mathrm{mg}, 3.55 \mathrm{mmol})$ and triethyl orthoformate $(8.2 \mathrm{~mL}, 49.65 \mathrm{mmol})$ was heated at reflux for $5 \mathrm{~h}$ at $145^{\circ} \mathrm{C}$. The solution was evaporated to dryness using a rotary evaporator. The residue was dissolved in formic acid $(99 \% ; 27 \mathrm{~mL})$, and the solution was heated at reflux at $110{ }^{\circ} \mathrm{C}$ for $5 \mathrm{~h}$. When the reaction was complete, the excess formic acid was removed using the rotary evaporator, and the residue was purified by column chromatography (dichloromethane/methanol, 97:3) to give $\mathbf{1 2}(540 \mathrm{mg}, 85 \%$ ) as a yellow solid. $R_{\mathrm{f}}=0.6$ (dichloromethane/methanol, 9:1). m.p. 232-234 ${ }^{\circ} \mathrm{C} .{ }^{1} \mathrm{H}$ NMR (300 MHz, [D 6 DMSO): $\delta=8.06$ (s, $1 \mathrm{H}, 2-$ $\mathrm{H}), 6.43(\mathrm{~s}, 1 \mathrm{H}, 7-\mathrm{H}), 3.99\left(\mathrm{~s}, 3 \mathrm{H}, \mathrm{OCH}_{3}\right), 3.85\left(\mathrm{~s}, 3 \mathrm{H}, \mathrm{OCH}_{3}\right)$ ppm. ${ }^{13} \mathrm{C}$ NMR (75 MHz, [D $]$ DMSO): $\delta=157.7$ (s, C-4, C-6), 152.1 (C-7a), 141.6 (C-2), 121.9 (C-3a), 83.7 (C-7), 52.5, 52.4 (2 $\left.\mathrm{OCH}_{3}\right)$ ppm. HRMS $\left(\mathrm{ESI}^{+}\right)$: calcd. for $\mathrm{C}_{8} \mathrm{H}_{9} \mathrm{~N}_{3} \mathrm{O}_{2}[\mathrm{M}+\mathrm{H}]^{+}$ 180.0767; found 180.0777.
4,6-Dimethoxy-9-(2,3,5-tri- $O$-acetyl- $\beta$-D-ribofuranosyl)-3-deazapurine (13a) and 4,6-Dimethoxy-7-(2,3,5-tri- $O$-acetyl- $\beta$-D-ribofuranosyl)-3-deazapurine (13b): TMSOTf $(1.22 \mathrm{~mL}, 6.70 \mathrm{mmol})$ was added to a stirred solution of compound $12(600 \mathrm{mg}, 3.35 \mathrm{mmol})$, sugar 9 (1.1 g, $3.68 \mathrm{mmol})$, and DBU $(0.56 \mathrm{~mL}, 3.68 \mathrm{mmol})$ in $\mathrm{CH}_{3} \mathrm{CN}(15 \mathrm{~mL})$ at $0{ }^{\circ} \mathrm{C}$. The mixture was stirred at $60^{\circ} \mathrm{C}$ for $12 \mathrm{~h}$, then it was poured into $\mathrm{NaHCO}_{3}$ (aq. solution). The mixture was extracted with ethyl acetate. The organic phase was washed with brine, dried $\left(\mathrm{MgSO}_{4}\right)$, and filtered, and the solvents were evaporated. The residue was purified by silica gel column chromatography (dichloromethane/methanol, 98:2) to give compound 13a $(740 \mathrm{mg}, 50 \%)$ as a pale yellow foam. $R_{\mathrm{f}}=0.3(n$-hexane/ethyl acetate, 1:1). ${ }^{1} \mathrm{H}$ NMR (500 MHz, $\mathrm{CDCl}_{3}$ ): $\delta=7.89$ (s, $\left.1 \mathrm{H}, 8-\mathrm{H}\right), 6.48$ (s, $1 \mathrm{H}, 3-\mathrm{H}), 5.94\left(\mathrm{~d}, J=5.70 \mathrm{~Hz}, 1 \mathrm{H}, 1^{\prime}-\mathrm{H}\right), 5.52(\mathrm{t}, J=5.70 \mathrm{~Hz}$, $\left.1 \mathrm{H}, 2^{\prime}-\mathrm{H}\right), 5.40\left(\mathrm{dd}, J=4.20,5.70 \mathrm{~Hz}, 1 \mathrm{H}, 3^{\prime}-\mathrm{H}\right), 4.11-4.46(\mathrm{~m}$, $\left.3 \mathrm{H}, 4^{\prime}-\mathrm{H}, 5 \mathrm{a}^{\prime}-\mathrm{H}, 5 \mathrm{~b}^{\prime}-\mathrm{H}\right), 4.11\left(\mathrm{~s}, 3 \mathrm{H}, \mathrm{OCH}_{3}\right), 3.94\left(\mathrm{~s}, 3 \mathrm{H}, \mathrm{OCH}_{3}\right)$, $2.21\left(\mathrm{~s}, 3 \mathrm{H}, \mathrm{CH}_{3}\right), 2.15$ (s, $\left.3 \mathrm{H}, \mathrm{CH}_{3}\right), 2.08\left(\mathrm{~s}, 3 \mathrm{H}, \mathrm{CH}_{3}\right) \mathrm{ppm} .{ }^{13} \mathrm{C}$ NMR (125 MHz, $\left.\mathrm{CDCl}_{3}\right): \delta=170.5,169.7,169.3$ (3 C=O), 159.3 (C-6), 154.1 (C-2), 142.1 (C-4), 139.3 (C-8), 124.2 (C-5), 87.2 (C1'), 82.6 (C-3), 80.4 (C-4'), 72.9 (C-2'), 70.2 (C-3'), 62.9 (C-5'), 54.3, $53.7\left(2 \mathrm{OCH}_{3}\right), 20.9,20.6,20.4\left(3 \mathrm{CH}_{3}\right) \mathrm{ppm}$. HRMS $\left(\mathrm{ESI}^{+}\right)$: calcd. for $\mathrm{C}_{19} \mathrm{H}_{23} \mathrm{~N}_{3} \mathrm{O}_{9}[\mathrm{M}+\mathrm{H}]^{+}$438.1506; found 438.1506 .

Further elution (dichloromethane/MeOH, 97:3) gave 13b (310 mg, $32 \%$ ) as a pale yellow foam. $R_{\mathrm{f}}=0.31$ ( $n$-hexane/ethyl acetate, $\left.1: 1\right)$. ${ }^{1} \mathrm{H}$ NMR $\left(600 \mathrm{MHz}, \mathrm{CDCl}_{3}\right): \delta=8.04(\mathrm{~s}, 1 \mathrm{H}, 8-\mathrm{H}), 6.17$ (s, $1 \mathrm{H}$, $3-\mathrm{H}), 5.53\left(\mathrm{~d}, J=5.80 \mathrm{~Hz}, 1 \mathrm{H}, 1^{\prime}-\mathrm{H}\right), 5.52(\mathrm{t}, J=5.80 \mathrm{~Hz}, 1 \mathrm{H}$, $\left.2^{\prime}-\mathrm{H}\right), 5.40\left(\mathrm{t}, J=5.80 \mathrm{~Hz}, 1 \mathrm{H}, 3^{\prime}-\mathrm{H}\right), 4.16-4.26\left(\mathrm{~m}, 3 \mathrm{H}, 4^{\prime}-\mathrm{H}\right.$, $\left.5 \mathrm{a}^{\prime}-\mathrm{H}, 5 \mathrm{~b}^{\prime}-\mathrm{H}\right), 3.89$ (s, $\left.3 \mathrm{H}, \mathrm{OCH}_{3}\right), 3.73\left(\mathrm{~s}, 3 \mathrm{H}, \mathrm{OCH}_{3}\right), 1.97$ (s, $\left.3 \mathrm{H}, \mathrm{CH}_{3}\right), 1.96\left(\mathrm{~s}, 3 \mathrm{H}, \mathrm{CH}_{3}\right), 1.92\left(\mathrm{~s}, 3 \mathrm{H}, \mathrm{CH}_{3}\right) \mathrm{ppm} .{ }^{13} \mathrm{C} \mathrm{NMR}$ $\left(150 \mathrm{MHz}, \mathrm{CDCl}_{3}\right): \delta=169.9,169.1,168.7(3 \mathrm{C}=\mathrm{O}), 158.2(\mathrm{C}-6)$, 154.6 (C-2), 148.1 (C-4), 143.1 (C-8), 112.7 (C-5), 89.5 (C-3), 88.5 $\left(\mathrm{C}-1^{\prime}\right), 78.8\left(\mathrm{C}-4^{\prime}\right), 74.0\left(\mathrm{C}-2^{\prime}\right), 68.7\left(\mathrm{C}-3^{\prime}\right), 62.1\left(\mathrm{C}-5^{\prime}\right), 53.9,53.2$ $\left(2 \mathrm{OCH}_{3}\right), 20.3,20.0,20.0\left(3 \mathrm{CH}_{3}\right)$ ppm. HRMS $\left(\mathrm{ESI}^{+}\right)$: calcd. for $\mathrm{C}_{19} \mathrm{H}_{23} \mathrm{~N}_{3} \mathrm{O}_{9}[\mathrm{M}+\mathrm{H}]^{+}$438.1506; found 438.1505.

2,6-Dimethoxy-9-( $\beta$-D-ribofuranosyl)-3-deazapurine (14): Hydrazine $(80 \% ; 7 \mathrm{~mL})$ was added to a solution of $13 \mathbf{a}(200 \mathrm{mg}, 457 \mu \mathrm{mol})$ in ethanol $(1 \mathrm{~mL})$, and the mixture was heated at $100{ }^{\circ} \mathrm{C}$ for $12 \mathrm{~h}$. The reaction mixture was concentrated, and the residue was purified by column chromatography (dichloromethane/methanol, 9:1) to give $14(136 \mathrm{mg}, 95 \%)$ as a solid. $R_{\mathrm{f}}=0.5$ (dichloromethane/methanol, 9:1). ${ }^{1} \mathrm{H}$ NMR (500 MHz, $\left.\mathrm{CD}_{3} \mathrm{OD}\right): \delta=8.26$ (s, $\left.1 \mathrm{H}, 8-\mathrm{H}\right), 6.74$ (s, $1 \mathrm{H}, 3-\mathrm{H}), 5.77$ (d, $\left.J=6.20 \mathrm{~Hz}, 1 \mathrm{H}, 1^{\prime}-\mathrm{H}\right), 5.51$ (s, $\left.1 \mathrm{H}, \mathrm{OH}\right), 5.25$ (s, $1 \mathrm{H}, \mathrm{OH}), 5.16$ (s, $1 \mathrm{H}, \mathrm{OH}), 4.32$ (br. s, $\left.1 \mathrm{H}, 2^{\prime}-\mathrm{H}\right), 4.11$ (br. s, $\left.1 \mathrm{H}, 3^{\prime}-\mathrm{H}\right), 3.96-3.80\left(\mathrm{~m}, 4 \mathrm{H}, \mathrm{OCH}_{3}, 4^{\prime}-\mathrm{H}\right), 3.85\left(\mathrm{~s}, 3 \mathrm{H}, \mathrm{OCH}_{3}\right)$, 3.50-3.68 (m, $\left.2 \mathrm{H}, 5 \mathrm{a}^{\prime}-\mathrm{H}, 5 \mathrm{~b}^{\prime}-\mathrm{H}\right)$ ppm. ${ }^{13} \mathrm{C}$ NMR $(125 \mathrm{MHz}$, $\left.\mathrm{CD}_{3} \mathrm{OD}\right): \delta=158.0$ (C-6), 153.0 (C-2), 142.7 (C-4), 141.5 (C-8), 123.5 (C-5), $88.8\left(\mathrm{C}-1^{\prime}\right), 85.7$ (C-3), $83.0\left(\mathrm{C}-4^{\prime}\right), 73.6\left(\mathrm{C}-2^{\prime}\right), 70.1$ $\left(\mathrm{C}-3^{\prime}\right), 61.2\left(\mathrm{C}-5^{\prime}\right), 53.9,53.0\left(2 \mathrm{OCH}_{3}\right)$ ppm. HRMS (ESI $\left.{ }^{+}\right)$: calcd. for $\mathrm{C}_{13} \mathrm{H}_{17} \mathrm{~N}_{3} \mathrm{O}_{6}[\mathrm{M}+\mathrm{H}]^{+} 312.1190$; found 312.1194.

2-(Allyloxy)-6-methoxy-3-nitropyridin-4-amine (15): Sodium allyl alkoxide ( $5 \mathrm{M}$ in allyl alcohol; $5 \mathrm{~mL}, 25.1 \mathrm{mmol}$ ) was added to a solution of $10(1.0 \mathrm{~g}, 5.2 \mathrm{mmol})$ in allyl alcohol $(25 \mathrm{~mL})$, and the mixture was heated at $60^{\circ} \mathrm{C}$ for $1.5 \mathrm{~h}$. The mixture was concentrated, the residue was dissolved in ethyl acetate and washed with water $(10 \mathrm{~mL})$. The organic layer was was dried $\left(\mathrm{MgSO}_{4}\right)$ and concentrated. The residue was purified by column chromatography ( $n$ hexane/ethyl acetate, 9:1) to give $\mathbf{1 5}(805 \mathrm{mg}, 82 \%)$ as a yellow solid. $R_{\mathrm{f}}=0.3$ ( $n$-hexane/ethyl acetate, 8:2). m.p. $68-70{ }^{\circ} \mathrm{C} .{ }^{1} \mathrm{H}$ NMR $\left(500 \mathrm{MHz}, \mathrm{CDCl}_{3}\right): \delta=6.29$ (br. s, $\left.2 \mathrm{H}, \mathrm{NH}_{2}\right), 5.95-6.01(\mathrm{~m}, 1 \mathrm{H}$, $\mathrm{CH}=\mathrm{C}), 5.63(\mathrm{~s}, 1 \mathrm{H}, 5-\mathrm{H}), 5.43(\mathrm{dd}, J=1.52,17.2 \mathrm{~Hz}, 1 \mathrm{H}$, $\left.\mathrm{CH}_{2}=\mathrm{C}\right), 5.22\left(\mathrm{dd}, J=1.52,10.30 \mathrm{~Hz}, 1 \mathrm{H}, \mathrm{CH}_{2}=\mathrm{C}\right), 4.85-4.93(\mathrm{~m}$, $\left.2 \mathrm{H}, \mathrm{CH}_{2}\right), 3.83\left(\mathrm{~s}, 3 \mathrm{H}, \mathrm{OCH}_{3}\right) \mathrm{ppm} .{ }^{13} \mathrm{C} \mathrm{NMR}(125 \mathrm{MHz}$, 
$\left.\mathrm{CDCl}_{3}\right): \delta=162.6(\mathrm{C}-6), 157.9(\mathrm{C}-4), 152.8(\mathrm{C}-2), 131.4(\mathrm{CH}=\mathrm{C})$, $116.8(\mathrm{~s}, \mathrm{C}=\mathrm{CH}, \mathrm{C}-3), 86.3(\mathrm{C}-5), 66.8\left(\mathrm{CH}_{2}\right), 53.0\left(\mathrm{OCH}_{3}\right) \mathrm{ppm}$. HRMS $\left(\mathrm{ESI}^{+}\right)$: calcd. for $\mathrm{C}_{9} \mathrm{H}_{11} \mathrm{~N}_{3} \mathrm{O}_{4}[\mathrm{M}+\mathrm{H}]^{+}$226.0822; found 226.0818 .

4-Amino-6-methoxy-3-nitropyridin-2-ol (16): Compound $\mathbf{1 5}$ (800 mg, $3.55 \mathrm{mmol})$ was dissolved in dry dichloromethane $(25 \mathrm{~mL})$, and tetrakis(triphenylphosphine)palladium $(0)$ (40 mg, $35 \mu \mathrm{mol})$ and 1,3-dimethylbarbituric acid ( $832 \mathrm{mg}, 5.33 \mathrm{mmol})$ were added simultaneously. The reaction mixture was stirred at room temp for $2.5 \mathrm{~h}$. The resulting precipitate was obtained by filtration, and it was washed with cold dichloromethane to give $\mathbf{1 6}$ (540 mg, 98\%) as a yellow solid. $R_{\mathrm{f}}=0.21$ (ethyl acetate). m.p. 241-243 ${ }^{\circ} \mathrm{C} .{ }^{1} \mathrm{H}$ NMR (600 MHz, [D 6 DMSO): $\delta=11.40(\mathrm{~s}, 1 \mathrm{H}$, $\mathrm{OH}), 8.24$ (br. s, $\left.2 \mathrm{H}, \mathrm{NH}_{2}\right), 5.34$ (s, $\left.1 \mathrm{H}, 5-\mathrm{H}\right), 3.79$ (s, $\left.3 \mathrm{H}, \mathrm{OCH}_{3}\right)$ ppm. ${ }^{13} \mathrm{C}$ NMR $\left(150 \mathrm{MHz},\left[\mathrm{D}_{6}\right] \mathrm{DMSO}\right): \delta=158.6,157.1,156.6$ (C-4, C-2, C-6), 115.6 (C-3), 75.4 (C-5), $56.2\left(\mathrm{OCH}_{3}\right)$ ppm. HRMS $\left(\mathrm{ESI}^{+}\right)$: calcd. for $\mathrm{C}_{6} \mathrm{H}_{7} \mathrm{~N}_{3} \mathrm{O}_{4}\left[\mathrm{M}+\mathrm{H}^{+}\right.$186.0509; found 186.0507 .

2-Chloro-6-methoxy-3-nitropyridin-4-amine (17): A solution of 16 (400 mg, $2.16 \mathrm{mmol})$ in $\mathrm{POCl}_{3}(2.5 \mathrm{~mL})$ was heated at $95^{\circ} \mathrm{C}$ for $4 \mathrm{~h}$. Then the reaction mixture was allowed to cool down to room temperature, and the $\mathrm{POCl}_{3}$ was evaporated. The residue was dissolved in ethyl acetate, and this solution was washed with $\mathrm{NaHCO}_{3}$ (saturated aq.; $2 \times 5 \mathrm{~mL}$ ). The organic phase was dried with $\mathrm{MgSO}_{4}$ and concentrated. The oily residue was purified by silica gel column chromatography (dichloromethane) to give 17 (290 mg, $78 \%)$ as a yellow solid. $R_{\mathrm{f}}=0.35$ ( $n$-hexane/ethyl acetate, 7:3). m.p. 140-142 ${ }^{\circ} \mathrm{C} .{ }^{1} \mathrm{H}$ NMR (500 MHz, [D $]$ DMSO): $\delta=6.11$ (s, $1 \mathrm{H}, 5-$ $\mathrm{H}), 3.81$ (s, $\left.3 \mathrm{H}, \mathrm{OCH}_{3}\right) \mathrm{ppm} .{ }^{13} \mathrm{C}$ NMR $\left(125 \mathrm{MHz},\left[\mathrm{D}_{6}\right] \mathrm{DMSO}\right)$ : $\delta=162.5,151.7,141.8$ (C-4, C-2, C-6), 127.8 (C-3), 92.1 (C-5), 54.3 $\left(\mathrm{OCH}_{3}\right)$ ppm. HRMS $\left(\mathrm{ESI}^{+}\right)$: calcd. for $\mathrm{C}_{6} \mathrm{H}_{6} \mathrm{ClN}_{3} \mathrm{O}_{3}[\mathrm{M}+\mathrm{H}]^{+}$ 204.0170; found 204.0172.

4-Chloro-6-methoxy-1H-imidazo[4,5-c|pyridine (18): A solution of sodium dithionite in water $(10 \mathrm{~mL})$ was added to a solution of $\mathbf{1 7}$ (400 mg, $1.96 \mathrm{mmol})$ in ethanol $(10 \mathrm{~mL})$, and the mixture was stirred at room temperature for $15 \mathrm{~min}$. The mixture was concentrated, the residue was dissolved in ethyl acetate and washed with water $(5 \mathrm{~mL})$. The organic layer was dried with $\mathrm{MgSO}_{4}$ and concentrated. The crude oily 3,4-diaminopyridine was used in the next step without further purification. A mixture of crude 3,4-diaminopyridine (300 $\mathrm{mg}, 1.73 \mathrm{mmol}$ ) and triethyl orthoformate $(4 \mathrm{~mL}, 24.19 \mathrm{mmol})$ was heated at reflux at $145^{\circ} \mathrm{C}$ for $5 \mathrm{~h}$. The solution was evaporated to dryness using a rotary evaporator. The residue was dissolved in formic acid $(99 \% ; 4 \mathrm{~mL})$ and the solution was heated at reflux at $110^{\circ} \mathrm{C}$ for $5 \mathrm{~h}$. When the reaction was complete, the excess formic acid was removed using a rotary evaporator. The residue was purified by column chromatography to give $\mathbf{1 8}$ $(216 \mathrm{mg}, 68 \%)$ as a yellow solid. $R_{\mathrm{f}}=0.15(n$-hexane/ethyl acetate, 4:6). m.p. $233-235^{\circ} \mathrm{C} .{ }^{1} \mathrm{H}$ NMR (300 MHz, [D 6 DMSO): $\delta=8.31$ (s, $1 \mathrm{H}, 2-\mathrm{H}), 6.87(\mathrm{~s}, 1 \mathrm{H}, 7-\mathrm{H}), 3.86\left(\mathrm{OCH}_{3}\right) \mathrm{ppm} .{ }^{13} \mathrm{C} \mathrm{NMR}$ (75 MHz, [D 6 ]DMSO): $\delta=158.3$ (C-6), 145.1 (C-2), 143.8, 135.6, 132.2 (C-7a, C-3a, C-4, assigned by HMBC), 90.5 (C-7), 54.3 $\left(\mathrm{OCH}_{3}\right)$ ppm. HRMS $\left(\mathrm{ESI}^{+}\right)$: calcd. for $\mathrm{C}_{7} \mathrm{H}_{6} \mathrm{ClN}_{3} \mathrm{O}[\mathrm{M}+\mathrm{H}]^{+}$ 184.0272; found 184.0278.

6-Chloro-2-methoxy-9-(2,3,5-tri- $\boldsymbol{O}$-acetyl- $\beta$-D-ribofuranosyl)-3deazapurine (19): TMSOTf $(0.89 \mathrm{~mL}, 4.99 \mathrm{mmol})$ was added to a stirred solution of compound $17(450 \mathrm{mg}, 2.45 \mathrm{mmol})$, sugar 9 (858 mg, $2.70 \mathrm{mmol})$, and DBU $(0.41 \mathrm{~mL}, 2.70 \mathrm{mmol})$ in $\mathrm{CH}_{3} \mathrm{CN}$ $(12 \mathrm{~mL})$ at $0{ }^{\circ} \mathrm{C}$. The mixture was stirred at $60^{\circ} \mathrm{C}$ for $12 \mathrm{~h}$, then it was poured into $\mathrm{NaHCO}_{3}$ (aq. solution). The mixture was extracted with ethyl acetate $(3 \times)$. The combined organic extracts were washed with brine, dried $\left(\mathrm{MgSO}_{4}\right)$, and filtered, and the solvents were evaporated. The residue was purified by silica gel col- umn chromatography ( $n$-hexane/ethyl acetate, 6:4) to give compound $19(783 \mathrm{mg}, 74 \%)$ as a thick yellow liquid. $R_{\mathrm{f}}=0.27(n-$ hexane/ethyl acetate, 1:1). ${ }^{1} \mathrm{H}$ NMR $\left(600 \mathrm{MHz}, \mathrm{CDCl}_{3}\right): \delta=8.05$ (s, $1 \mathrm{H}, 8-\mathrm{H}), 6.89(\mathrm{~s}, 1 \mathrm{H}, 3-\mathrm{H}), 5.96\left(\mathrm{~d}, J=5.80 \mathrm{~Hz}, 1 \mathrm{H}, 1^{\prime}-\mathrm{H}\right)$, $5.50\left(\mathrm{~d}, J=5.80 \mathrm{~Hz}, 1 \mathrm{H}, 2^{\prime}-\mathrm{H}\right), 5.40(\mathrm{dd}, J=3.93,5.80 \mathrm{~Hz}, 1 \mathrm{H}$, $\left.3^{\prime}-\mathrm{H}\right), 4.31-4.49$ (m, $\left.3 \mathrm{H}, 4^{\prime}-\mathrm{H}, 5 \mathrm{a}^{\prime}-\mathrm{H}, 5 \mathrm{~b}^{\prime}-\mathrm{H}\right), 3.98$ (s, $3 \mathrm{H}, \mathrm{OCH}_{3}$ ), $2.23\left(\mathrm{~s}, 3 \mathrm{H}, \mathrm{CH}_{3}\right), 2.16$ (s, $\left.3 \mathrm{H}, \mathrm{CH}_{3}\right), 2.09\left(\mathrm{~s}, 3 \mathrm{H}, \mathrm{CH}_{3}\right) \mathrm{ppm} .{ }^{13} \mathrm{C}$ NMR $\left(150 \mathrm{MHz}, \mathrm{CDCl}_{3}\right): \delta=170.4,169.7,169.4(3 \mathrm{C}=\mathrm{O}), 159.7$ (C-2), 142.3 (C-8), 141.6 (C-6), 139.0 (C-4), 134.4 (C-5), 90.1 (C3), $87.4\left(\mathrm{C}-1^{\prime}\right), 80.6\left(\mathrm{C}-4^{\prime}\right), 72.7\left(\mathrm{C}-2^{\prime}\right), 70.0\left(\mathrm{C}-3^{\prime}\right), 62.8\left(\mathrm{C}-5^{\prime}\right)$, $54.8\left(\mathrm{OCH}_{3}\right), 20.9,20.6,20.4\left(3 \mathrm{CH}_{3}\right)$ ppm. HRMS (ESI $\left.{ }^{+}\right)$: calcd. for $\mathrm{C}_{18} \mathrm{H}_{20} \mathrm{ClN}_{3} \mathrm{O}_{8}[\mathrm{M}+\mathrm{H}]^{+}$442.1011; found 442.1012.

6-Amino-2-methoxy-9-( $\beta$-D-ribofuranosyl)-3-deazapurine (2): Hydrazine $(80 \% ; 10 \mathrm{~mL})$ was added to a solution of $19(340 \mathrm{mg}$, $769 \mu \mathrm{mol})$ in ethanol $(1 \mathrm{~mL})$, and the mixture was heated at $100^{\circ} \mathrm{C}$ for $2 \mathrm{~h}$. The reaction mixture was concentrated, Raney Ni $(50 \%$; $10 \mathrm{~mL}$ ) was added, and the mixture was heated at $100^{\circ} \mathrm{C}$ for $2 \mathrm{~h}$. The reaction mixture was filtered through Celite, the filtrate was concentrated, and the residue was purified by column chromatography (dichloromethane/methanol, 9:1) to give $2(149 \mathrm{mg}, 65 \%)$ as a thick liquid. $R_{\mathrm{f}}=0.25$ (ethyl acetate/methanol, 6:1). ${ }^{1} \mathrm{H}$ NMR (500 MHz, $\left.\mathrm{CD}_{3} \mathrm{OD}\right): \delta=8.19$ (s, $\left.1 \mathrm{H}, 8-\mathrm{H}\right), 6.38$ (s, $\left.1 \mathrm{H}, 3-\mathrm{H}\right), 5.80$ (d, $\left.J=5.80 \mathrm{~Hz}, 1 \mathrm{H}, 1^{\prime}-\mathrm{H}\right), 4.44\left(\mathrm{dd}, J=4.11,5.80 \mathrm{~Hz}, 1 \mathrm{H}, 2^{\prime}-\right.$ $\mathrm{H}), 4.26\left(\mathrm{dd}, J=4.11,5.80 \mathrm{~Hz}, 1 \mathrm{H}, 3^{\prime}-\mathrm{H}\right), 4.11(\mathrm{~d}, J=4.11 \mathrm{~Hz}$, $\left.1 \mathrm{H}, 4^{\prime}-\mathrm{H}\right), 3.70-3.90\left(\mathrm{~m}, 5 \mathrm{H}, \mathrm{OCH}_{3}, 5 \mathrm{a}^{\prime}-\mathrm{H}, 5 \mathrm{~b}^{\prime}-\mathrm{H}\right) \mathrm{ppm} .{ }^{13} \mathrm{C}$ NMR (125 MHz, CD $\left.{ }_{3} \mathrm{OD}\right): \delta=161.9(\mathrm{C}-2), 150.8(\mathrm{C}-6), 142.7$ (C-

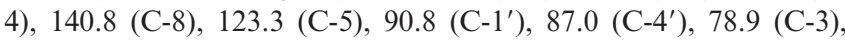
$75.6\left(\mathrm{C}-2^{\prime}\right), 71.7\left(\mathrm{C}-3^{\prime}\right), 62.6\left(\mathrm{C}-5^{\prime}\right), 55.0\left(\mathrm{OCH}_{3}\right)$ ppm. HRMS $\left(\mathrm{ESI}^{+}\right)$: calcd. for $\mathrm{C}_{12} \mathrm{H}_{16} \mathrm{~N}_{4} \mathrm{O}_{5}[\mathrm{M}+\mathrm{H}]^{+}$297.1193; found 297.1194.

Supporting Information (see footnote on the first page of this article): ${ }^{1} \mathrm{H}$ and ${ }^{13} \mathrm{C}$ NMR spectra.

\section{Acknowledgments}

We are grateful to Prof. Jef Rozenski for recording HRMS spectra, Luc Baudemprez for NMR experiments, and Mrs. Chantal Biernaux for excellent editorial help. We are indebted to Dr. Jang and Dr. Bel Abed for helpful discussions.

[1] P. Herdewijn, Modified Nucleosides in Biochemistry, Biotechnology and Medicine, John Wiley \& Sons, 2008.

[2] E. Ichikawa, K. Kato, Curr. Med. Chem. 2001, 8, 385-423.

[3] D. G. Bartholomew, P. Dea, R. K. Robins, G. R. Revankar, J. Org. Chem. 1975, 40, 3708-3713.

[4] J. A. Montgomery, H. J. Thomas, Adv. Carbohydr. Chem. 1962, 17, 301-369.

[5] F. Seela, S. Lampe, Helv. Chim. Acta 1991, 74, 1790-1800.

[6] P. C. Zamecnik, Biochem. J. 1962, 85, 257-264.

[7] C. Woenckha, G. Pfleider, Biochem. Z. 1965, 341, 495-499.

[8] W. Saenger, Angew. Chem. 1973, 85, 680-690; Angew. Chem. Int. Ed. Engl. 1973, 12, 591-601.

[9] Y. M. Yutilov in Advances in Heterocyclic Chemistry, vol. 89 (Ed.: A. R. Katritzky), Elsevier Academic Press Inc, San Diego, 2005, pp. 159-270.

[10] K. V. Bukhryakov, A. V. Kurkin, M. A. Yurovskaya, Chem. Heterocycl. Compd. 2011, 47, 533-557.

[11] A. D. Khoje, C. Charnock, B. Wan, S. Franzblau, L.-L. Gundersen, Bioorg. Med. Chem. 2011, 19, 3483-3491.

[12] J. Zemlicka, S. Zhou, J. C. Drach, Wayne State University; The Regents of the University of Michigan, US 08232275, 2012.

[13] P. Naus, M. Kuchar, M. Hocek, Collect. Czech. Chem. Commun. 2008, 73, 665-678.

[14] J. Medzihradsky, T. Zimmerman, G. Wolberg, G. Elion, Immunopharmacol. Immunotoxicol. 1982, 4, 29-41. 
[15] T. A. Krenitsky, J. L. Rideout, E. Y. Chao, G. W. Koszalka, F. Gurney, R. C. Crouch, N. K. Cohn, G. Wolberg, R. Vinegar, J. Med. Chem. 1986, 29, 138-143.

[16] J. A. Montgome, K. Hewson, J. Med. Chem. 1966, 9, 105-107.

[17] R. T. Bartlett, A. F. Cook, M. J. Holman, W. W. McComas, E. F. Nowoswait, M. S. Poonian, J. A. Baird-Lambert, B. A. Baldo, J. F. Marwood, J. Med. Chem. 1981, 24, 947-954.

[18] S. S. Stokes, M. Gowravaram, H. Huynh, M. Lu, G. B. Mullen, B. Chen, R. Albert, T. J. O'Shea, M. T. Rooney, H. Hu, J. V. Newman, S. D. Mills, Bioorg. Med. Chem. Lett. 2012, 22, 8589.

[19] S. S. Stokes, H. Huynh, M. Gowravaram, R. Albert, M. Cavero-Tomas, B. Chen, J. Harang, J. T. Loch Iii, M. Lu, G. B. Mullen, S. Zhao, C.-F. Liu, S. D. Mills, Bioorg. Med. Chem. Lett. 2011, 21, 4556-4560.

[20] W. Bergmann, D. C. Burke, J. Org. Chem. 1956, 21, 226-228.

[21] P. A. Searle, T. F. Molinski, J. Nat. Prod. 1994, 57, 1452-1454.

[22] L. M. Ojha, R. Pratap, D. S. Bhakuni, Indian J. Chem. Sect. B: Org. Chem. Incl. Med. Chem. 1994, 33, 140.

[23] L. M. Ojha, D. Gulati, N. Seth, D. S. Bhakuni, R. Pratap, K. C. Agarwal, Nucleosides Nucleotides 1995, 14, 1889-1900.

[24] R. Rickles, L. Pierce, M. S. Lee, WO 2009011897, 2009.

[25] P. Richardson, WO 2004078184, 2004.

[26] E. D. Savory (Cambridge Biotechnology Ltd., UK), WO 2005054269, 2005.

[27] S. A. Benner, Acc. Chem. Res. 2004, 37, 784-797.
[28] I. Hirao, M. Kimoto, R. Yamashige, Acc. Chem. Res. 2012, 45, 2055-2065.

[29] M. Jeselnik, S. Jaksa, J. Kobe, Croat. Chem. Acta 2004, 77, 153-160.

[30] A. Stimac, I. Leban, J. Kobe, Synlett 1999, 1069-1073.

[31] P. Herdewijn, J. Balzarini, M. Baba, R. Pauwels, A. Van Aerschot, G. Janssen, E. De Clercq, J. Med. Chem. 1988, 31, 20402048.

[32] P. Herdewijn, Antisense Nucleic Acid Drug Dev. 2000, 10, $297-$ 310

[33] R. Cosstick, X. Li, D. K. Tuli, D. M. Williams, B. A. Connolly, P. C. Newman, Nucleic Acids Res. 1990, 18, 4771-4778.

[34] K. L. Seley, P. I. O’Daniel, S. Salim, Nucleosides Nucleotides Nucleic Acids 2003, 22, 2133-2144.

[35] M. J. Kim, Synth. Commun. 2010, 40, 2988-2999.

[36] J. A. Montgomery, A. T. Shortnacy, S. D. Clayton, J. Heterocycl. Chem. 1977, 14, 195-197.

[37] S. Ninkovic, J. F. Braganza, M. R. Collins, J. C. Kath, H. Li, D. T. Richter (Pfizer Inc., USA), WO 2010016005, 2010.

[38] A. B. Eldrup, M. Prhavc, J. Brooks, B. Bhat, T. P. Prakash, Q. Song, S. Bera, N. Bhat, P. Dande, P. D. Cook, C. F. Bennett, S. S. Carroll, R. G. Ball, M. Bosserman, C. Burlein, L. F. Colwell, J. F. Fay, O. A. Flores, K. Getty, R. L. LaFemina, J. Leone, M. MacCoss, D. R. McMasters, J. E. Tomassini, D. von Langen, B. Wolanski, D. B. Olsen, J. Med. Chem. 2004, 47, 5284-5297.

[39] H. Tsukamoto, T. Suzuki, Y. Kondo, Synlett 2007, 3131-3136. Received: August 26, 2013 Published Online: November 22, 2013 